ACCEPTED MANUSCRIPT

\title{
Controlled Fabrication of Gold Nanotip Arrays by Nanomolding-Necking Technology
}

To cite this article before publication: Guoxing Han et al 2019 Nanotechnology in press https://doi.org/10.1088/1361-6528/ab62cb

\section{Manuscript version: Accepted Manuscript}

Accepted Manuscript is "the version of the article accepted for publication including all changes made as a result of the peer review process, and which may also include the addition to the article by IOP Publishing of a header, an article ID, a cover sheet and/or an 'Accepted

Manuscript' watermark, but excluding any other editing, typesetting or other changes made by IOP Publishing and/or its licensors"

This Accepted Manuscript is @ 2019 IOP Publishing Ltd.

During the embargo period (the 12 month period from the publication of the Version of Record of this article), the Accepted Manuscript is fully protected by copyright and cannot be reused or reposted elsewhere.

As the Version of Record of this article is going to be / has been published on a subscription basis, this Accepted Manuscript is available for reuse under a CC BY-NC-ND 3.0 licence after the 12 month embargo period.

After the embargo period, everyone is permitted to use copy and redistribute this article for non-commercial purposes only, provided that they adhere to all the terms of the licence https://creativecommons.org/licences/by-nc-nd/3.0

Although reasonable endeavours have been taken to obtain all necessary permissions from third parties to include their copyrighted content within this article, their full citation and copyright line may not be present in this Accepted Manuscript version. Before using any content from this article, please refer to the Version of Record on IOPscience once published for full citation and copyright details, as permissions will likely be required. All third party content is fully copyright protected, unless specifically stated otherwise in the figure caption in the Version of Record.

View the article online for updates and enhancements. 


\title{
Controlled Fabrication of Gold Nanotips Arrays by Nanomolding-Necking Technology
}

Guoxing $\mathrm{Han}^{1}$, Yupeng $\mathrm{Wu}^{1}$, Weidong Yan ${ }^{1}$, Langquan Shui ${ }^{1}$, Xiangzheng Jia ${ }^{1}$, Enlai $\mathrm{Gao}^{1, *}$, Minqiang Jiang ${ }^{2,3}$, and Ze Liu ${ }^{1,2, *}$

${ }^{1}$ Department of Engineering Mechanics, School of Civil Engineering, Wuhan University, Wuhan, Hubei 430072, China.

${ }^{2}$ State Key Laboratory of Nonlinear Mechanics, Institute of Mechanics, Chinese Academy of Sciences, Beijing 100190, China.

${ }^{3}$ School of Engineering Science, University of Chinese Academy of Sciences, Beijing 101408, China.

*Corresponding authors. Email: enlaigao@whu.edu.cn; ze.liu@whu.edu.cn

\begin{abstract}
The fabrication of nanotips has been driven by the increasing industrial demands in developing high-performance multifunctional nanodevices. In this work, we proposed a controlled, rapid as well as low cost nanomolding-necking technology to fabricate gold nanotips arrays. The geometries of gold nanotips with cone angle range of $\sim 28-84^{\circ}$ and curvature radii of $<5 \mathrm{~nm}$ can be prepared by tailoring the diameters of raw nanorods in nanomolding process or modulating the necking temperature. Molecular dynamics simulation reveals that the formation of the nanotip geometries are determined by the interplay between dislocation-based and diffusion-based deformation mechanisms, intrinsically arising from the nonlinear dependence of atom diffusion on temperature and sample size. The strong controllability, mass production and low-cost of the developed nanomolding-necking technology make it highly promising in developing nanodevices for a wide range of applications, such as probing, sensing, antireflection coating and nanoindentation.
\end{abstract}

Keywords: nanomolding-necking; nanotips; controlled fabrication. 


\section{Introduction}

Metal nanomaterials can be divided into zero-, one-, or two-dimensional $(0,1,2 \mathrm{D})$ structures. 1D nanostructures, consisting of rods, wires, tips, ribbons, and pores, possess fantastic properties benefiting from their quite unique structures, which make them promising for a large number of applications, such as electronic circuits, nanostencil lithography, reinforcement phase of composites, bio-and chemical sensors [1]. For example, Deng et al. recently developed controllable methods of wet etching to fabricate nanopore arrays with tunable shapes for nanostencil lithography [2, 3]. Nanotip, as one of the most intriguing members of 1D nanostructures, has been widely used in cold cathode field emission [4], scanning probe microscopy [5], antireflection coating [6] and nanoindentation [7]. To this end, considerable efforts have been made in developing nanotip fabrication technologies, which can be divided into top-down and bottom-up strategies [8], such as etching techniques [2,3], electron beam-induced deposition [9], laser ablation [10], physical vapor deposition [11], chemical vapor deposition [12], electron cyclotron resonance plasma process [13] etc. However, these methods are usually limited for mass production by their poor-controllability, complex processes or high cost. Therefore, a controlled, rapid, and low-cost nanotip fabrication technology is still highly desirable.

Necking, in engineering or materials science, refers to a tensile deformation mode of ductile materials where relatively large amounts of strain localize in a small region [14]. As a common deformation and failure of material, necking has been widely used in the deformation control of rigid plastics $[15,16]$, large deformation of polypropylene [17-20], fabrication of glass wire or tube [21] and metallic glasses [22, 23], and preparation of high quality semiconductor metals [24]. However, the necking phenomenon has been rarely used in nanofabrication considering the challenging of preparing and manipulating small-scale specimens. Recently we demonstrate a lowcost fabrication technology of nanorod arrays, named as superplastic nanomolding (SPNM), which can fabricate nanorods arrays through either dislocation slip or atom diffusion mechanisms [25-27]. The advantage of this SPNM technology is that 
nanorods arrays with varying diameters can be fabricated with strong controllability.

Combing the fabrication of nanorod arrays by SPNM and the subsequently shaping nanorods into nanotips by necking deformation, this work proposes a new strategy to fabricate gold nanotips arrays. It is found experimentally that lowertemperature necking of nanorods with larger diameters facilitates the formation of sharper nanotips. The effects of sample size and necking temperature were systematically investigated by molecular dynamics (MD) simulations, which accords remarkably well with the experiments and reveals that the nanotip geometries are determined by the competition between dislocation-based and diffusion-based deformations.

\section{Materials and Methods}

\subsection{Fabrication of nanorod arrays by superplastic nanomolding}

The fabrication of gold nanotips arrays consists of two steps, SPNM of nanorods arrays as well as necking of the nanorods into nanotips, as illustrated in Figure 1a-d. In the first step, stainless steel thin plate, ultra-thin anodic aluminum oxide template (AAO template, produced by Shenzhen Topological Seminal Membrane Technology Company) and gold plate (purity of 99.999\%, purchased from Hezheng New Material Company) were stacked as sandwich structures (Figure 1a). The sandwich sample was put between two parallel plates of a universal testing machine, which is equipped with a home-built heater. When the sample was heated to a typical target temperature of 800 $\mathrm{K}$, the metal/mold combination was compressed at a loading rate of $0.5 \mathrm{~mm} / \mathrm{min}$ (corresponding to a strain rate of $8 \times 10^{-3} \mathrm{~s}^{-1}$ ) until $500 \mathrm{MPa}$, and then held for $600 \mathrm{~s}$. The gold will fill into the AAO nanoholes through diffusion dominated creep deformation [25]. After fully filled into the AAO template, the gold nanorods will continuously grow to contact the stainless steel thin plate, and finally bond with the stainless steel through atom diffusion (Figure 1b). Thus, gold nanorods arrays with two ends fixed onto gold and stainless steel substrates, respectively, were obtained. 
AAO templates with the same diameter $(\sim 10 \mathrm{~mm})$ but different through-holes having diameters of $25 \mathrm{~nm}, 90 \mathrm{~nm}, 260 \mathrm{~nm}$, and heights of $120 \mathrm{~nm}, 400 \mathrm{~nm}, 1200 \mathrm{~nm}$, respectively, were used in the experiments. Therefore, the thickness of AAO template equals to the heights of through-holes therein. In our experiments, the effective nanomolding region is about $3 \mathrm{~mm}$ in diameter, in which the pulling-off force is about $80 \mathrm{~N}$ (Figure S1). The necking region is determined by the molded region. In principle, the gold nanotips could be uniform over large area (Figure S2). Because the fixing condition is very important in the fabricating of high-quality nanotips. Herein, we built a structure to clamp the stainless steel plate (with size much larger than the molded region), the induced in-plane stretching stress can thus help to reduce the out-of-plane deformation of the stainless steel plate (Figure S3). In this structure, the top end of the gold rod is clamped and fixed, the stacked AAO and stainless steel plates are fixed between an orifice plate and a solid plate by using bolts (Figure S3b).

(a)

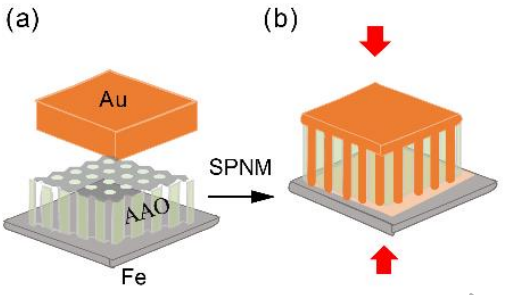

(b) area in (e).
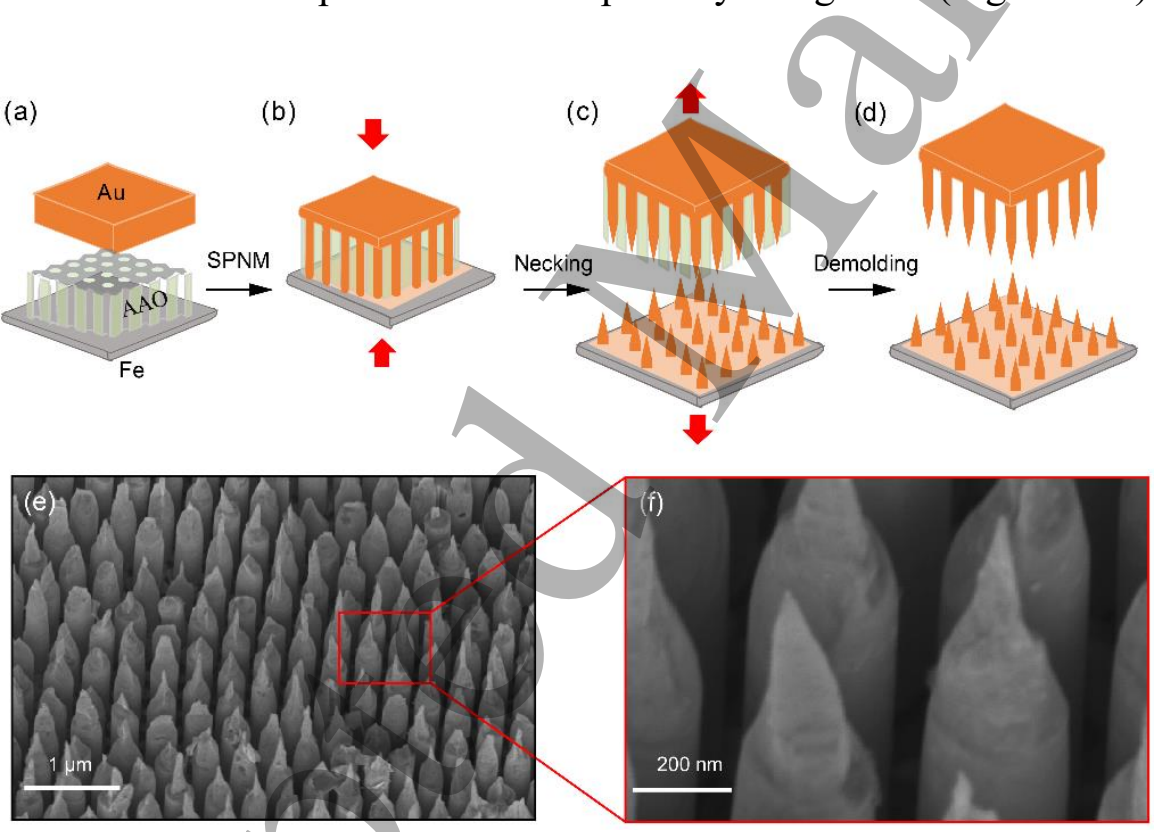

Figure 1. (a-d) Schematic illustration of nanomolding-necking technology. (e) Typical SEM images of gold nanotips arrays. These nanotips were obtained by necking 260$\mathrm{nm}$-diameter gold nanorod array at $300 \mathrm{~K}$. (f) Magnified SEM image of the selected red 


\subsection{Fabrication of nanotips by necking of the gold nanorod arrays}

In the second step, the fabricated gold nanorod array were cooled down to various of temperatures, and subsequently the as-formed sandwich structure was stretched along the axial direction of the nanorods by oppositely pulling the gold and stainless steel substrates. The loading rate was $0.5 \mathrm{~mm} / \mathrm{min}$ ( strain rate $8 \times 10^{-3} \mathrm{~s}^{-1}$ ). As the loading force increasing, the nanorod arrays firstly elastically stretched, then plastically deformed and finally necked to break, which formed gold nanotips arrays (Figure 1c). The processed structure was then immersed in a $\mathrm{KOH}$ solution (with concentration of $3 \mathrm{~mol} / \mathrm{L}$ and temperature of $300 \mathrm{~K}$ ) for 2 hours to remove the AAO template, the gold nanotips arrays attached on the surface of the gold and stainless steel substrates were obtained (Figure 1d).

\subsection{Measurement of nanotip geometries}

To accurately characterize the geometries of the prepared gold nanotips, two generatrixes of the circular truncated cone were extended to intersect in a single point, and the angle between these lines is defined as the cone angle of a nanotip. Specifically, we use a field emission scanning electron microscopy (SEM, Zeiss Sigma 500) to measure the cone angle of the nanotips. The prepared sample with nanotips attached was placed onto the sample stage of the SEM. The sample stage was tilted by $60^{\circ}$ to get a good view of the nanotips. Since the gold nanotips are perpendicular to the gold substrate and hence the sample stage, the angle between the electron beam and the nanotip will be also $60^{\circ}$. Therefore, the actual cone angle of a nanotip $(\alpha)$ can be calculated based on the measured cone angle $(\beta)$ by the geometric conversion $[26,28]$

$$
\alpha=\tan ^{-1}\left(\frac{\sqrt{3}}{2} \tan \beta\right)
$$

\subsection{Molecular dynamics simulation}

MD simulations were carried out using large-scale atomic/molecular massively parallel simulator (LAMMPS) computational package [29]. The optimized embedded atom 
method (EAM) potential is adopted for its high fidelity and robustness [30]. The Newton equations of motion are integrated using the Verlet algorithm with a time step of 1 fs. Circular-cross-section gold nanorods were created out of an ideal centered cubic crystal with cross-section orientations of [100]. All the samples are modelled with length of $50 \mathrm{~nm}$ but varying diameters from 6 to $10 \mathrm{~nm}$. Before the tensile deformation protocol, the prepared samples were first fully relaxed to an equilibrium configuration using a conjugated-gradient algorithm and then thermally equilibrated to target temperatures $(300 \mathrm{~K}$ and $800 \mathrm{~K})$ for 100 ps using a Nosé-Hoover thermostat with a characteristic relaxation time of $0.1 \mathrm{ps}$. Afterwards, the uniaxial tensile strain with a strain rate of $3 \times 10^{7} \mathrm{~s}^{-1}$ was applied by deforming the periodic simulation box in the [100] direction until failure of structures. Finally, the formed nanotips were equilibrated at the target temperatures for $100 \mathrm{ps}$ to track their geometry evolution.

\section{Results and Discussion}

\subsection{Characterization of the prepared gold nanotips by nanomolding-necking} technology

Figure 1e-f shows a typical morphology of the prepared gold nanotips arrays characterized by SEM. A gold rod with diameter of $3 \mathrm{~mm}$ and height of $1 \mathrm{~cm}$ was stacked between a 10-mm-diameter AAO template, which has through nanoholes with diameter of $260 \mathrm{~nm}$ and heights of $1200 \mathrm{~nm}$, and a stainless steel plate with thickness of $50 \mu \mathrm{m}$. The processing conditions for nanomolding and necking are described in the materials and methods section. Because of the constraints between nanorods and AAO template during the stretching process, the necking region of nanorods usually occurs near the roots of the nanorods. Thus the average height of the nanotips arrays is equal to the thickness of AAO template and generally uniform. The necking randomly occurs nearing the roots of nanorods, which can be attributed to the stress concentration at the roots for the nucleation of necking. It is needed to point out that the shapes of gold nanotips on both the stainless steel substrate and gold substrate are observed similar (Figure S4). Additionally, high-aspect-ratio tips called 'rocket tips' [31] can be prepared 
by simply using AAO templates with high-aspect-ratio nanopores in our method. The uniformity of the prepared gold nanotips arrays on a large scale (Figure 1e) indicates the high efficiency and mass production of our method. Especially, by zooming-in the nanotips (Figure 1f), it is amazing to find that the curvature radii of the nanotips were smaller than $5 \mathrm{~nm}$. Such a sharp tip is highly desirable for the applications in tipenhanced sensors as well as high-resolution imaging in scanning probe microscopy.

\subsection{Modulating the geometries of nanotips by necking nanorods with different} diameters

The properties of nanorods are highly related to their specific surface area [32]. To investigate the surface effect on the nanotip geometries, we used 10-mm-diameter AAO templates having through-holes with diameter of $25 \mathrm{~nm}, 90 \mathrm{~nm}, 260 \mathrm{~nm}$, and height of $120 \mathrm{~nm}, 400 \mathrm{~nm}, 1200 \mathrm{~nm}$, respectively, from which nanorods with different diameters were prepared by SPNM. After necking the nanorods arrays at $300 \mathrm{~K}$ (see materials and methods section for details), gold nanotips arrays with different geometries were prepared (Figure 2a-c), where the cone angles of the nanotips were measured and calculated by using eq. (1) as $67^{\circ}, 56^{\circ}$ and $35^{\circ}$ for the nanorods with diameter of 22,86 and $260 \mathrm{~nm}$, respectively. By measuring at least 10 nanotips for each size, the averaged parameters of nanotip geometries were obtained. It is clear that as the diameter of nanorods increased from $25 \pm 3 \mathrm{~nm}, 90 \pm 5 \mathrm{~nm}$ to $268 \pm 28 \mathrm{~nm}$, the cone angle decreases from $69 \pm 7^{\circ}, 54 \pm 3^{\circ}$ to $32 \pm 6^{\circ}$, respectively. We can herein conclude that the larger nanorod diameter will result in the sharper of the nanotips. By further comparing the morphologies among the nanotips fabricated from nanorods with varying diameters, we observed that the length of necking zone increases with the diameter of nanorods (Figure 2a-c). However, it is noted that the curvature radii of the nanotips are almost insensitive to the diameters of the nanorods under these conditions, which are less than $5 \mathrm{~nm}$ for all the samples (Figure 2a-c). 

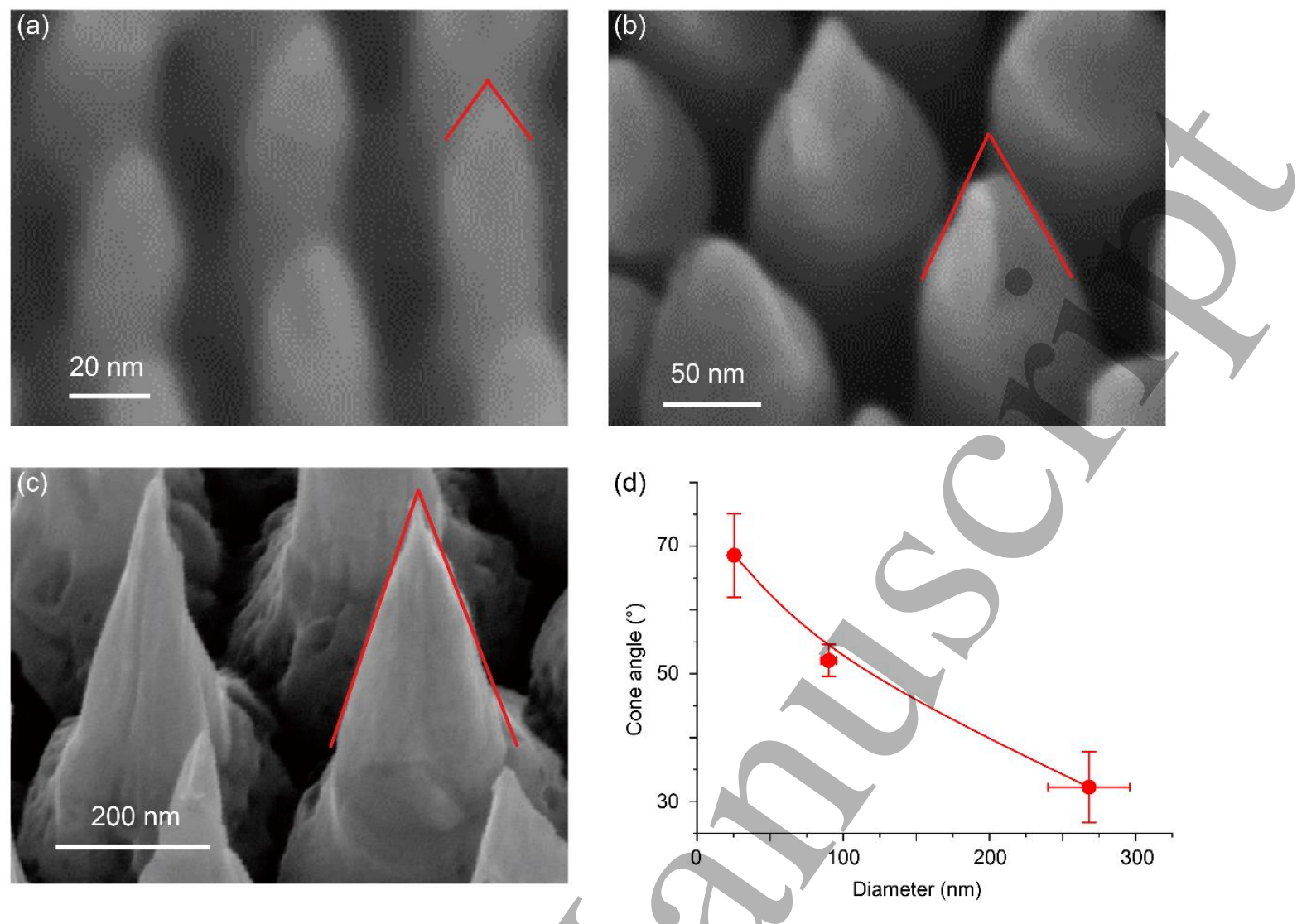

Figure 2. SEM images of the fabricated nanotips having diameters of (a) $22 \mathrm{~nm}$, (b) 86 $\mathrm{nm}$, and (c) $260 \mathrm{~nm}$. (d) The relationship between the cone angles and the diameters of nanorods.

\subsection{Modulating the geometries of nanotips by controlling the necking temperatures}

To investigate the temperature effect on the geometries of gold nanotips, the necking of gold nanorods arrays was carried out at different temperatures. To avoid the coupling with the effect of nanorod size as investigated above, the AAO template with the same diameter of $260 \mathrm{~nm}$ was used for all the samples, while three necking temperatures (300 $\mathrm{K}, 500 \mathrm{~K}$ and $800 \mathrm{~K}$ ) were chosen. The fabricated nanotips are shown in Figure 3a-c. Again, we measured and calculated the cone angles of the nanotips and the results are summarized in Figure 3d. We observed that the lower necking temperature leads to the sharper nanotips. Specifically, the cone angles of nanotips prepared at $300 \mathrm{~K}, 500 \mathrm{~K}$, and $800 \mathrm{~K}$ are $28 \pm 6^{\circ}, 66 \pm 8^{\circ}$ to $84 \pm 6^{\circ}$, respectively (Figure $3 \mathrm{~d}$ ), indicating the wide 
tunability of nanotip geometries by modulating necking temperature.
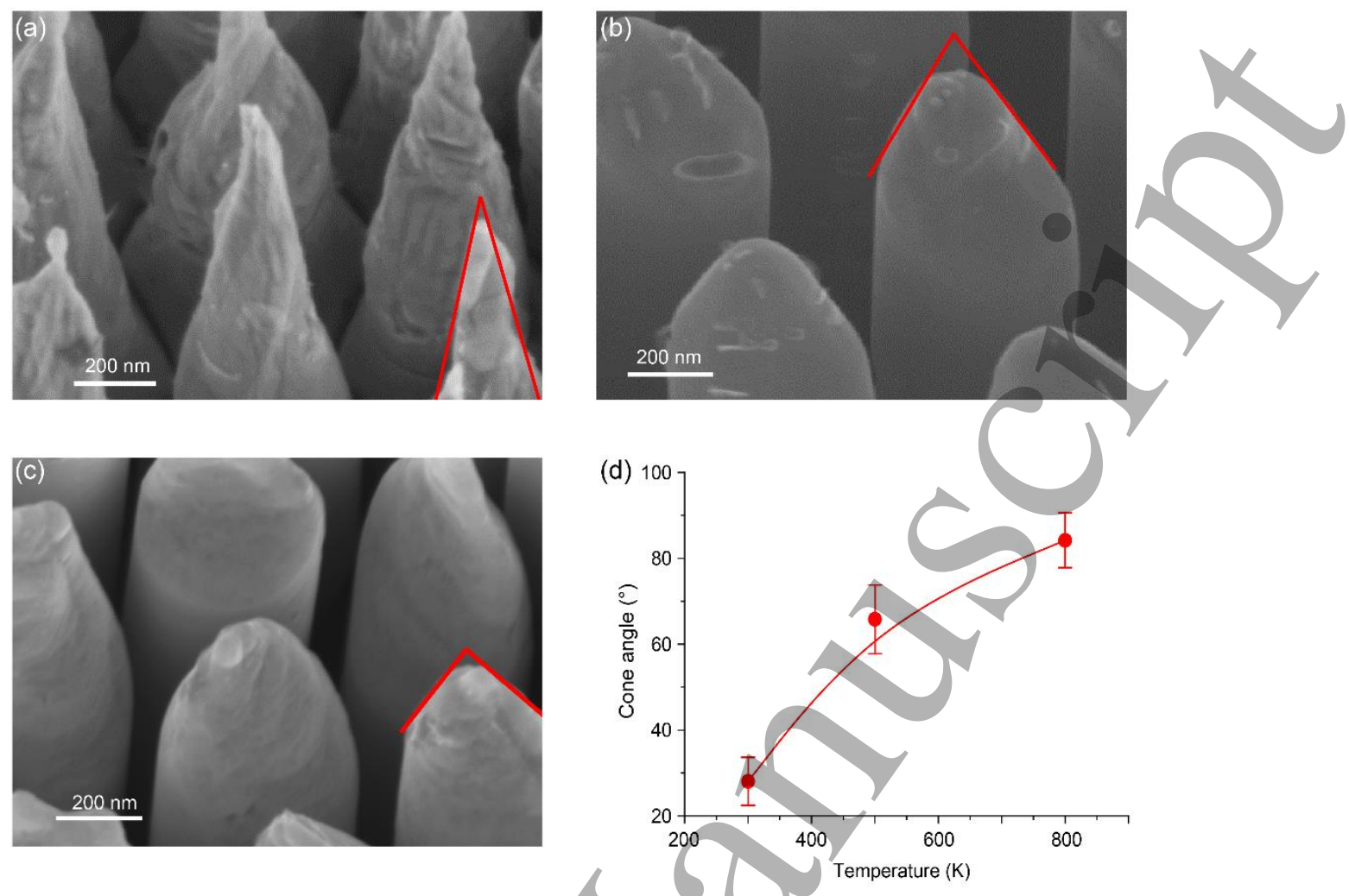

Figure 3. SEM images of nanotips obtained by necking 260 -nm-diameter nanorods at temperatures of (a) $300 \mathrm{~K}$, (b) $500 \mathrm{~K}$ and (c) $800 \mathrm{~K}$. (d) The relationship between the cone angles and the processing temperatures.

\subsection{Mechanism discussions}

To understand the underlying mechanism of the observed influence of nanorod diameters and necking temperatures on the sharpness of nanotips, MD simulations of nanorods with different diameters stretched at varying temperatures were conducted (see materials and methods section for details). The results are summarized in Figure 4. It can be found that the nanorods experience clearly necking process and thus develop into atom-thick nanotips. The geometries of gold nanorods with larger diameter evaluated more gently in wide regions and thus formed sharper nanotips. Meanwhile, the tips become blunt with the increasing of processing temperature. This evolutional trend of nanotips modulated by sample sizes and processing temperature agrees well 
with our experimental observation, which can be explained by the interplay between dislocation and atom diffusion based deformation. The dislocation-dominated deformation in the necking (strain localization) process is critical to create atomic steps and thus form sharp nanotips, while the diffusion helps to smooth out atomic steps in the tip surface and thus reduce the sharpness of nanotips. The diffusion coefficient for the atom diffusion can be given as

$D \propto v e^{-\Delta E / k_{\mathrm{B}} T}$

where $v$ and $\Delta E$ are the vibrational frequency of atoms and the potential energy barrier to diffusion, respectively, $T$ is temperature, and $k_{\mathrm{B}}$ is the Boltzmann constant. As shown in Figure 4, the potential energy of surface atoms is generally higher than that of bulk atoms, indicating their high activity (low $\Delta E$ ) that leads to fast atom diffusion (high $D$ ). The specific area in nanorods with smaller diameters is larger, making the diffusion based deformation mode arises and thus blunting the tips. Similarly, the increasing of processing temperature (high $k_{\mathrm{B}} T$ ) will also induce fast atom diffusion (high $D$ ) and thus blunt the tips. In general, as the nanorods have small diameters or are processed at high temperatures, the gold atoms at the surface, especially at the top surface of nanotips diffuse easily towards the base of nanotips to minimize the system energy, and thus form nanotips with large cone angles. Otherwise, as the dislocation-based deformation dominates the formation of nanotip geometries, the continuous dislocation slip is apt to render the sharp tips (Figure 4).

As discussed previously, the curvature radii of nanotips can be affected by the necking temperature and the specific area of samples. In addition, the uniformity of the prepared nanotips arrays may limit its wide applications. To solve this problem, ordered AAO templates with good uniformity should be used for fabricating uniform nanorods. Fortunately, there are some literatures reporting the preparation of uniform AAO templates by adding a pre-stamping process $[33,34]$. In addition to improving the uniformity of nanorods, the necking of nanorods also influences the top shape and height of each nanotip, which closely relates to the necking temperature and defects of 
nanorods. Although elevating the necking temperature can improve the uniformity, but the curvature of the top shape becomes larger because of the fast atom diffusion. Thus to improve the uniformity of nanotips, future efforts should be directed, but not limited, at fabricating defect-less nanorods and necking the nanorods under proper processing conditions.

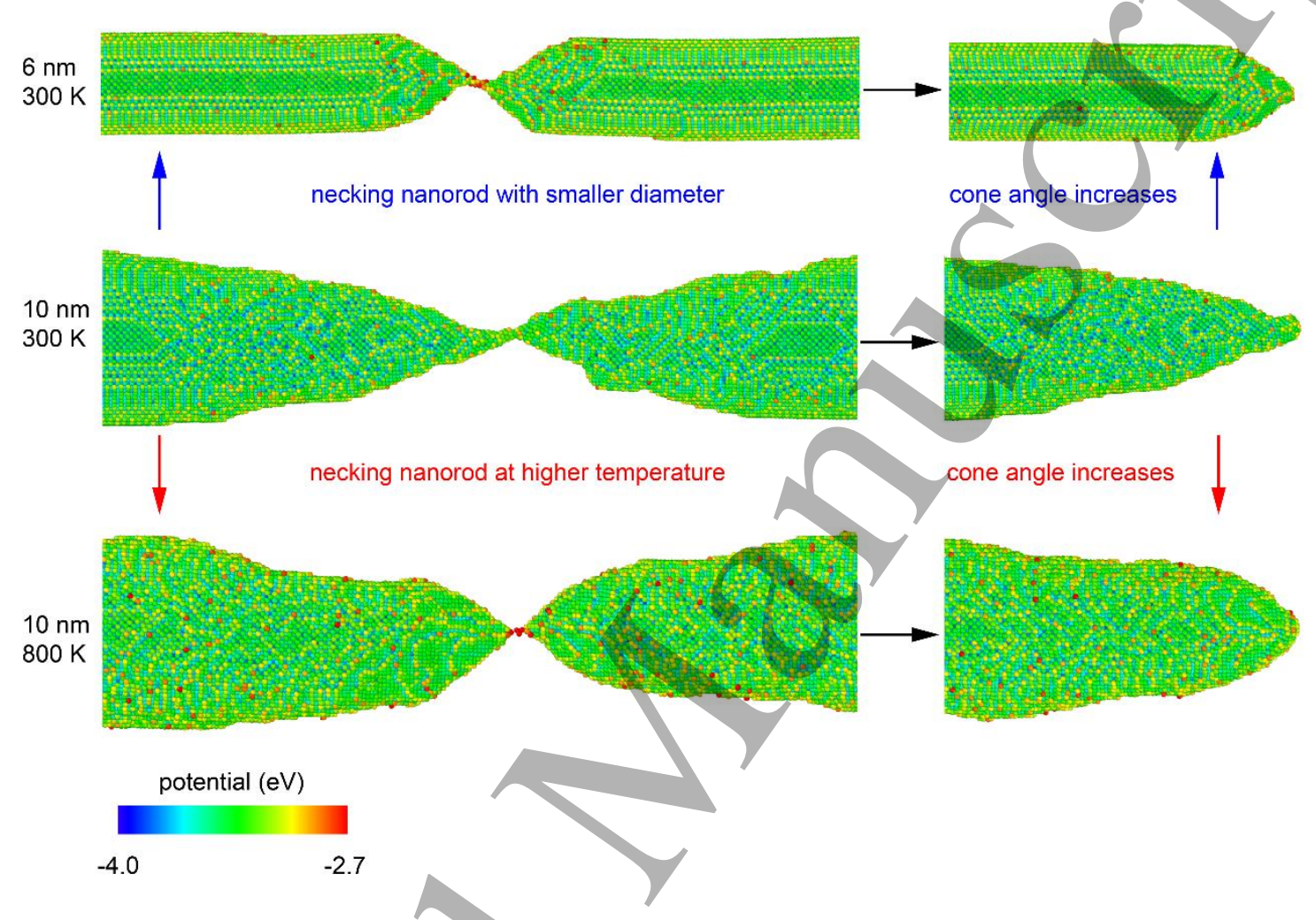

Figure 4. Snapshots of the nanorods with varying diameters $(6 \mathrm{~nm}$ and $10 \mathrm{~nm})$ stretched at different temperatures $(300 \mathrm{~K}$ and $800 \mathrm{~K}$ ) before breaking and after breaking with equilibrium for $100 \mathrm{ps}$. Atoms are colored by their values of potential energy.

Additionally, we remark here that there are some limits on MD simulations. The strain rate $\left(3 \times 10^{7}\right)$ in the simulations is much higher than that used in experiments $\left(8 \times 10^{-3} \mathrm{~s}^{-1}\right)$. The high strain rate is expected to reduce the time for atom diffusion in the necking and thus helps to form sharper nanotips, which results in some discrepancies between the MD simulations and the experiments. For example, the cone angle of 10nm-diamter nanotips observed in simulation is $30^{\circ}$ (necking at $300 \mathrm{~K}$, Figure 4), while the cone angle of $22-\mathrm{nm}$-diamter nanotips prepared at $300 \mathrm{~K}$ from experiments is much larger $\left(70^{\circ}\right.$, Figure $\left.2 \mathrm{a}\right)$. Despite these discrepancies, the MD simulations captured the 
evolutional trend of nanotip morphologies that are modulated by the effects of surface and temperature on atom diffusion, which agrees well with the experimental observations.

\section{Conclusion}

In summary, based on the developed nanomolding-necking technology, gold nanotips with controlled cone angles (from $28^{\circ}$ to $\left.84^{\circ}\right)$ and extremely small curvature radii $(<5$ $\mathrm{nm})$ were fabricated. It is found that the nanorod diameter and the necking temperature play important roles in the shaping of nanotips. By using MD simulations, the underlying mechanism is revealed as the competition between atom diffusion and dislocation based deformation mechanisms. These findings not only pave a way to study the deformation mechanism in metal nanorods, but also provide a practical technology for the mass fabrication of metal nanotips with strong controllability, easy scalability as well as low-cost. 


\section{Acknowledgements}

This work was supported by the Opening fund of State Key Laboratory of Nonlinear Mechanics, the National Natural Science Foundation of China (11902225, 11872284 , 11632009, and 11602175), Wuhan Science and Technology Bureau (2019010701011390), and the Fundamental Research Funds for the Central Universities (413000091). 


\section{References}

[1] Xia Y, Yang P, Sun Y, Wu Y, Mayers B, Gates B, Yin Y, Kim F and Yan H 2003 One-dimensional nanostructures: Synthesis, characterization, and applications Advanced Materials 15 353-389

[2] Deng T, Wang Y F, Chen Q, Chen H J and Liu Z W 2016 Massive fabrication of silicon nanopore arrays with tunable shapes Applied Surface Science 390 681-688

[3] Deng T, Li M W, Chen J, Wang Y F and Liu Z W 2014 Controllable fabrication of pyramidal silicon nanopore arrays and nanoslits for nanostencil lithography Journal of Physical Chemistry C 118 18110-18115

[4] Houdellier F, Masseboeuf A, Monthioux M and Hytch M J 2012 New carbon cone nanotip for use in a highly coherent cold field emission electron microscope Carbon 50 2037-2044

[5] Rezeq M d and Joachim C 2010 Scanning Probe Microscopy World Scientific

[6] Southwell W H 1991 Pyramid-array surface-relief structures producing antireflection index matching on optical-surfaces Journal of the Optical Society of America $A \mathbf{8}$ 549-553

[7] Kumaravelu G, Alkaisi M M, Bittar A, Macdonald D and Zhao J 2004 Damage studies in dry etched textured silicon surfaces Current Applied Physics 4 108-110

[8] Chattopadhyay S, Chen L and Chen K 2006 Nanotips: growth, model, and applications Critical Reviews in Solid State \& Materials Sciences 31 15-53

[9] Belova L M, Hellwig O, Dobisz E and Dan Dahlberg E 2012 Rapid preparation of electron beam induced deposition Co magnetic force microscopy tips with $10 \mathrm{~nm}$ spatial resolution Review of Scientific Instruments $\mathbf{8 3} 093711$

[10] Pedraza A J, Fowlkes J D and Lowndes D H 1999 Silicon microcolumn arrays grown by nanosecond pulsed-excimer laser irradiation Applied Physics Letters 74 2322-2324

[11] Wu Z S, Deng S Z, Xu N S, Chen J, Zhou J and Chen J 2002 Needle-shaped silicon carbide nanowires: Synthesis and field electron emission properties Applied Physics Letters 80 3829-3831

[12] Babu E S, Saravanakumar B, Ravi G, Yuvakkumar R, Ganesh V, Guduru R K and Kim S 2018 Zinc oxide nanotips growth by controlling vapor deposition on substrates Journal of Materials Science-Materials in Electronics 29 6149-6156

[13] Hsu C H, Lo H C, Chen C F, Wu C T, Hwang J S, Das D, Tsai J, Chen L C and Chen K H 2004 Generally applicable self-masked dry etching technique for nanotip array fabrication Nano Letters 4 471-475

[14] Bridgman P W 1952 Studies in large plastic flow and fracture McGraw-Hill New York

[15] Vincent P I 1960 The necking and cold-drawing of rigid plastics Polymer 1 7-19

[16] Georgievskii D 2015 Uniaxial extension of a thin rigid-plastic sheet in the presence of a neck Doklady Physics $60310-313$

[17] Sweeney J, Collins T L D, Coates P D and Ward I M 1997 Application of an elastic model to the large deformation, high temperature stretching of polypropylene 
Polymer 38 5991-5999

[18] Ibhadon A O 1990 Neck profiles in drawn polypropylene and ethylene propylene copolymers Makromolekulare Chemie-Macromolecular Chemistry and Physics, 191 1375-1382

[19] Liu T and Harrison I R 1987 The temperature rise on neck formation of polymers: Polypropylene and polyethylene Polymer 28 1860-1862

[20] Taylor W N and Clark E S 1978 Superdrawn filaments of polypropylene Polymer Engineering and Science 18 518-526

[21] Takayama S 1981 Fabrication of metallic-glass wire or tube by drawing Journal of Materials Science 16 269-271

[22] Hu Z, Meduri C S, Blawzdziewicz J and Kumar G 2019 Nanoshaping of glass forming metallic liquids by stretching: Evading lithography Nanotechnology $\mathbf{3 0}$ 075302

[23] Hasan M and Kumar G 2017 High-throughput drawing and testing of metallic glass nanostructures Nanoscale 9 3261-3268

[24] Langdo T A, Leitz C W, Currie M T, Fitzgerald E A, Lochtefeld A and Antoniadis D A 2000 High quality Ge on Si by epitaxial necking Applied Physics Letters 76 3700-3702

[25] Liu Z 2017 One-step fabrication of crystalline metal nanostructures by direct nanoimprinting below melting temperatures Nature Communications 814910

[26] Liu Z, Han G, Sohn S, Liu N and Schroers J 2019 Nanomolding of crystalline metals: The smaller the easier Physical Review Letters 122036101

[27] Liu Z 2019 Investigation of temperature and feature size effects on deformation of metals by superplastic nanomolding Physical Review Letters 122016101

[28] Rose D J 1956 On the magnification and resolution of the field emission electron microscope Journal of Applied Physics 27 215-220

[29] Plimpton S 1995 Fast parallel algorithms for short-range molecular-dynamics Journal of Computational Physics 117 1-19

[30] Sheng H W, Kramer M J, Cadien A, Fujita T and Chen M W 2011 Highly optimized embedded-atom-method potentials for fourteen FCC metals Physical Review B $\mathbf{8 3} 134118$

[31] Boisen A, Hansen O and Bouwstra S 1996 AFM probes with directly fabricated tips Journal of Micromechanics and Microengineering 6 58-62

[32] Park H S and Zimmerman J A 2005 Modeling inelasticity and failure in gold nanowires Physical Review B 72054106

[33] Li J J, Zhang W W, Song Y J, Yin W T and Zhang T 2016 Template transfer nanoimprint for uniform nanopores and nanopoles Journal of Nanomaterials 2016 $1-7$

[34] Alam K M, Singh A P, Bodepudi S C and Pramanik S 2011 Fabrication of hexagonally ordered nanopores in anodic alumina: An alternative pretreatment Surface Science 605 441-9 